\title{
TUBERCULOSIS OSTEOARTICULARES TRATADAS CON HIDRAZIDA DEL ACIDO ISONICOTINICO
}

\author{
Prof. EUGENIO DIAZ y Dr. MARIO GONZALEZ \\ Hospital "Roberto del Río".
}

En Mayo de 1952, basados en los trabajos de Elmendorf, quien demostró la notable difusibilidad de la hidrazida en el organismo, como asimismo su escasa acción tóxica, decidimos emplearla en el tratamiento de Ia tuberculosis osteoarticular. Posteriormente Bosworth, comunicó los resultados satisfactorios obtenjdos en estos enfermos con el empleo de la hidrazida. Al informar sobre los beneficios apreciables que poseía scbre el estado general, peso y apetito, recalcó la rápida mejoria de los trayectos fistulosos e insistió en los trastornos tóxicos secundarios, algunos de gravedad, que se presentaron al emplear el derivado isopropílico de la hidrazida.

ANALISIS DE LOS ENFERMOS

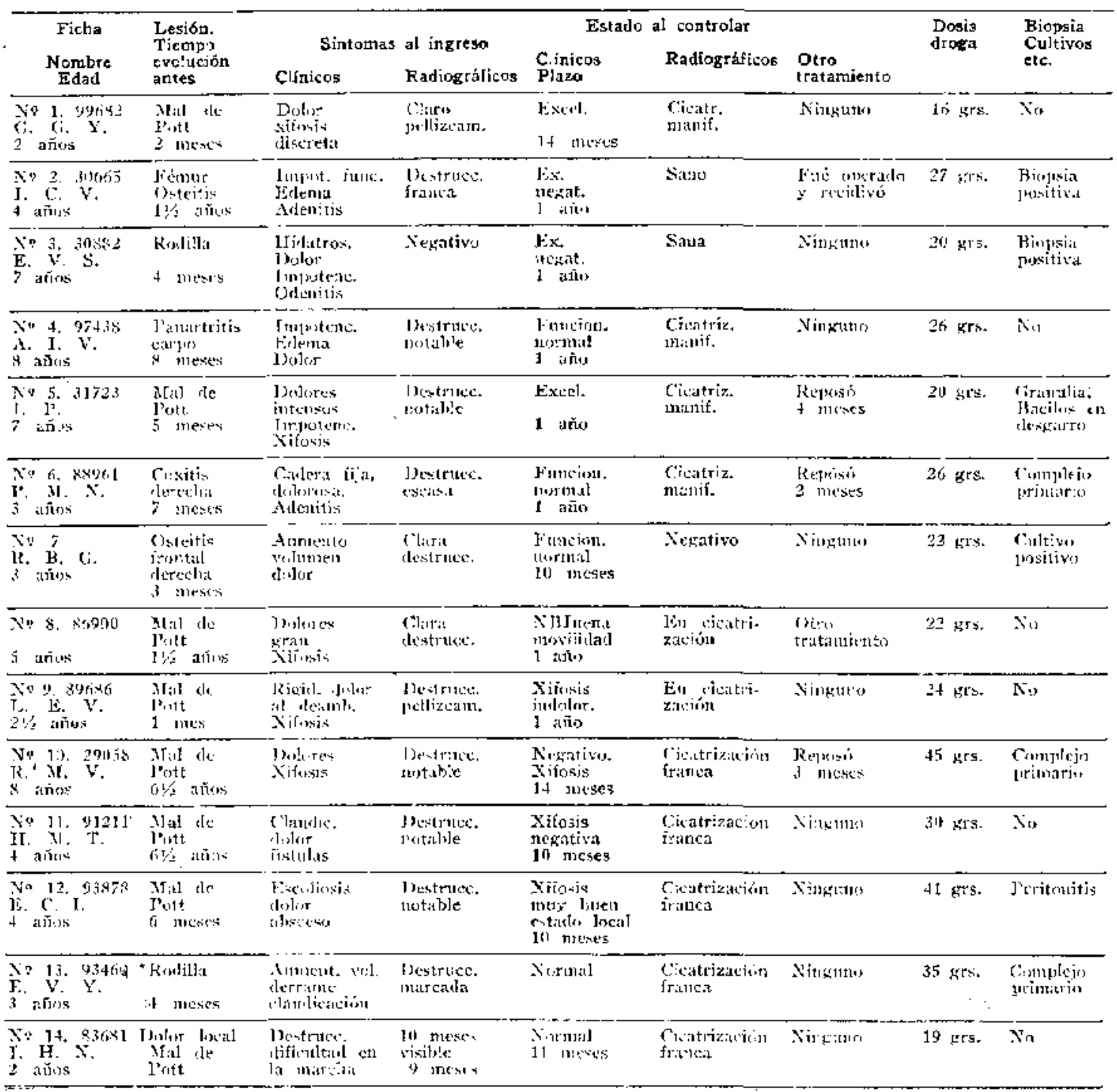


La tuberculosis ostearticular es bastante frecuente entre nosotros y requiere de tratamientos prolongados y costosos, cuyos resultados distan mucho de ser satisfactorios, ya que cuando los estimamos buenos, ello se hace a expensas de la pérdida de la función articular correspondiente. Sus complicaciones, unas derivadas de la enfermedad misma o de su tratamiento, dificultan la curación o dejan graves secuelas.

En esta comunicación preliminar daremos cuenta del plan de trabajo que nos trazamos y de sus resultados, que consideramos notablemente alentadores.

\section{ANÁLISIS DE LOS ENFERMOS}

Se escogieron tuberculosis óseas y osteoarticulares, de diferentes localizaciones, en su gran mayoría no sometidas anteriormente a ningún tratamiento. Reunimos asi, hasta la fecha, 72 casos en total, de los cuales sólo 14 enfermos llevan ya más de un año bajo nuestro control y a ellos nos referiremos especialmente en esta comunicación. Los datos principales referentes a su sintomatología, evolución y tratamiento, se resumen en el cuadro.
Se certificó el diagnóstico de tuberculosis, por la biepsia de la cápsula articular o del ganglio linfático correspondiente, en la mayoría de los casos. Además, se efectuó baciloscopía y despistaje de otras lesiones capaces de confirmar la etiología de la enfermedad.

En el cuadro adjunto pueden verse las edades, ya que se escogieron enfermos pequeños donde la gravedad del proceso es siempre mayor, especialmente en los Mal de Pott.

Se señalan especialmente el caso No 4 por tratarse de una panartritis de la mano, de franca gravedad, el caso $N^{\circ} 3$ por lo temprano del diagnóstico y los notables resultados obtenidos, los casos $N^{\circ} 5$ y 12 , en los que la gravedad de las lesiones médicas concomitantes, ensombrecían el pronóstico. En el primero de estos, existía una diseminación hematógena (véase la radiografía) con bacilosccpia positiva en el desgarro, gran hepato y esplenomegalia, tubérculos cutáneos y coroídeos, que ya a los tres meses de tratamiento estaban en franca regresión. También desaparecieron los dolores lumbares, de tipo radicular, que eran muy intensos. El segundo caso,

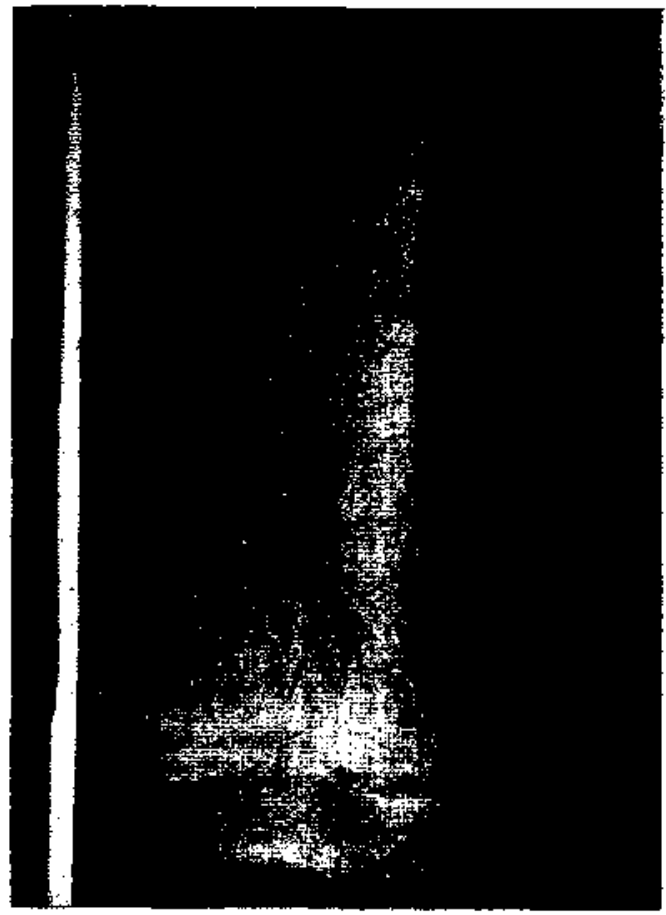

CASO No 3: Radiografia de rodila. A: Julio 1952. B: Agosto 1953. 


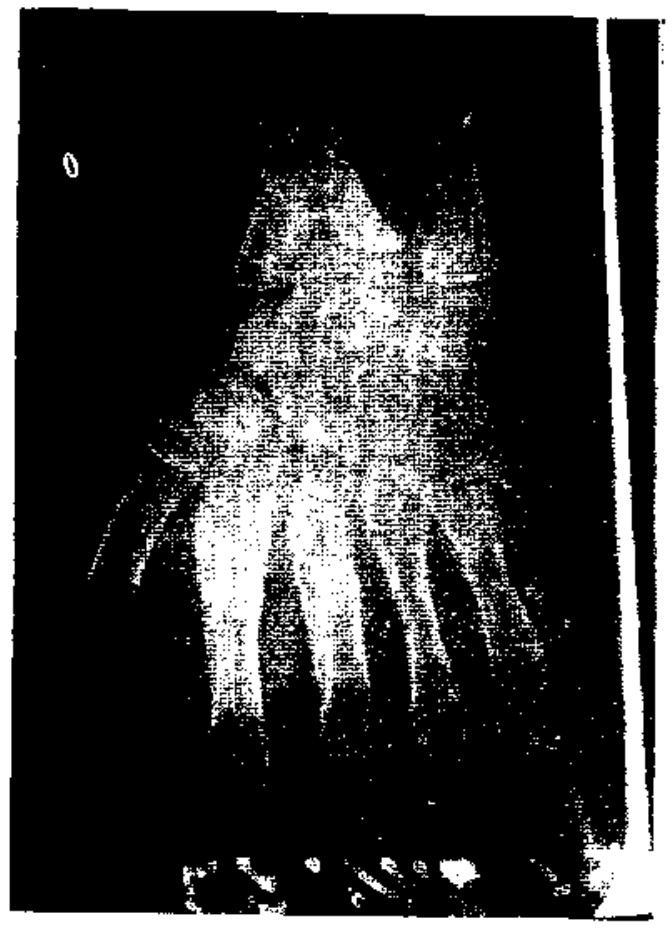

A

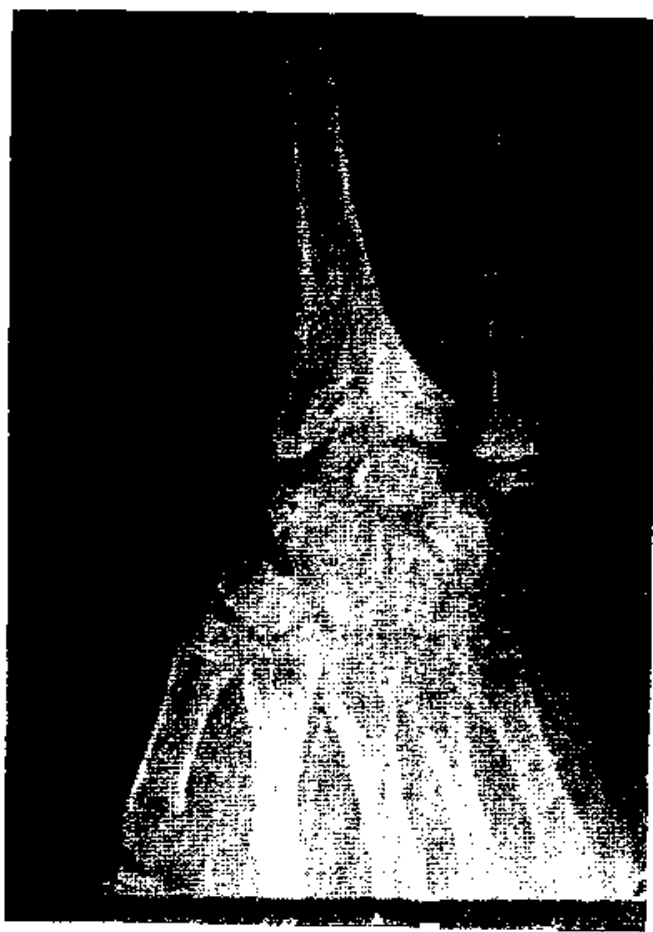

CASO N!' 4: Radiografia de carpo. A: Julio 1952. B: Agosto 1953.

presentó en los primeros días del tratanormal.

Controles: Se practicaron mensualmente los controles clínicos locales y generales y cada tres meses, radiografías, hemogramas y sedimentación.

\section{RESULTADOS}

A. Generales: Notable mejoria del apetito y peso, se observó en todos los enfermos. Las condiciones del estado gene- ra1, mejoraron aún en los más graves, demostrándose una franca mejoria de la toxemia tuberculosa. Sobre las lesiones derivadas de la generalización del bacilo, tales como la diseminación hematógena mencionada, los resultados concuerdan ampliamente con lo ya señalado por uno de nosotros en comunicación anterior. En cambio, no se observó modificación apreciable, en la evolución del complejo primario.
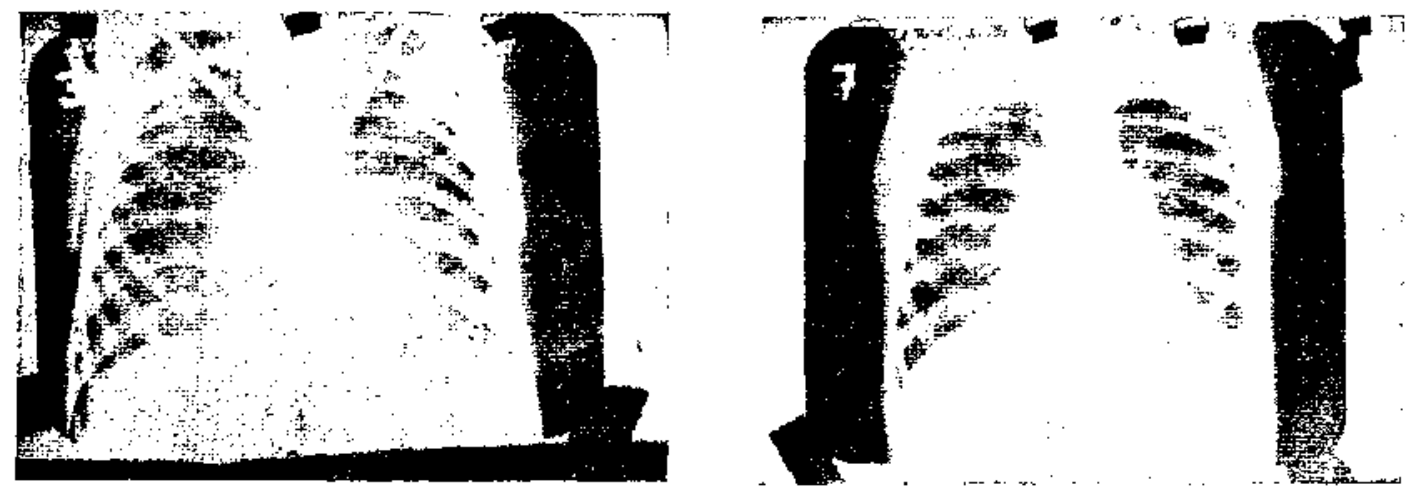

A

CASO No 5: Radiografia de tótax. A: Ottubre 1952. B: Mayo 1953. 


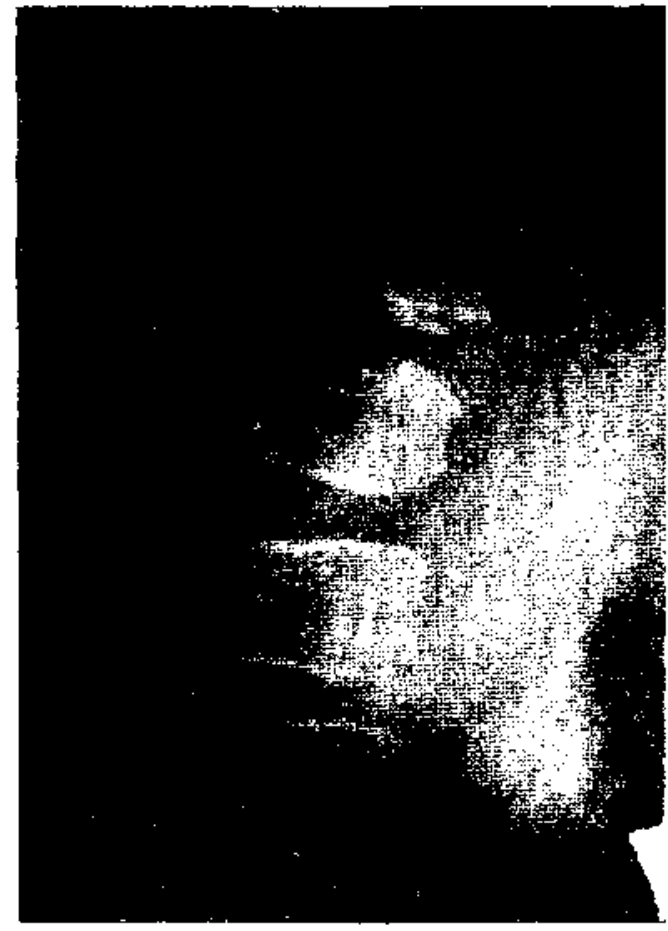

A

CASO $\Lambda^{4}$ 5: Radiogrália de columno

miento una peritonitis tuberculosa de forma ascitica, con pésimo estado general y ya a los dos meses de tratamiento no habian signos peritoneales y el $\mathrm{Mal}$ de Pot cesó en su sintomatología dolorosa.

\section{DOSIS DE LA DROGA}

Durante los primeros seis meses se dió 10 mgrs. por kilo de peso y en los seis meses siguientes se bajó a 5 mgrs. Nos parece, hasta el momento, que la cura de un año es suficiente. La tolerancia fú perfecta en todos los casos, no habiéndose observado efectos tóxicos de ninguna especie.

\section{OTROS TRATAMIENTOS MÉDICOS, QUIRÚRGICOS U ORTOPÉDICOS}

No se realizó ninguno. Cada enfermo guardó reposo en cama sólo mientras tuvo molestias, después pasó a hacer vida

Otra expresión de la mejoría de las condiciones generales, se reflejó en los resultados del análisis del cuadro hematológico.

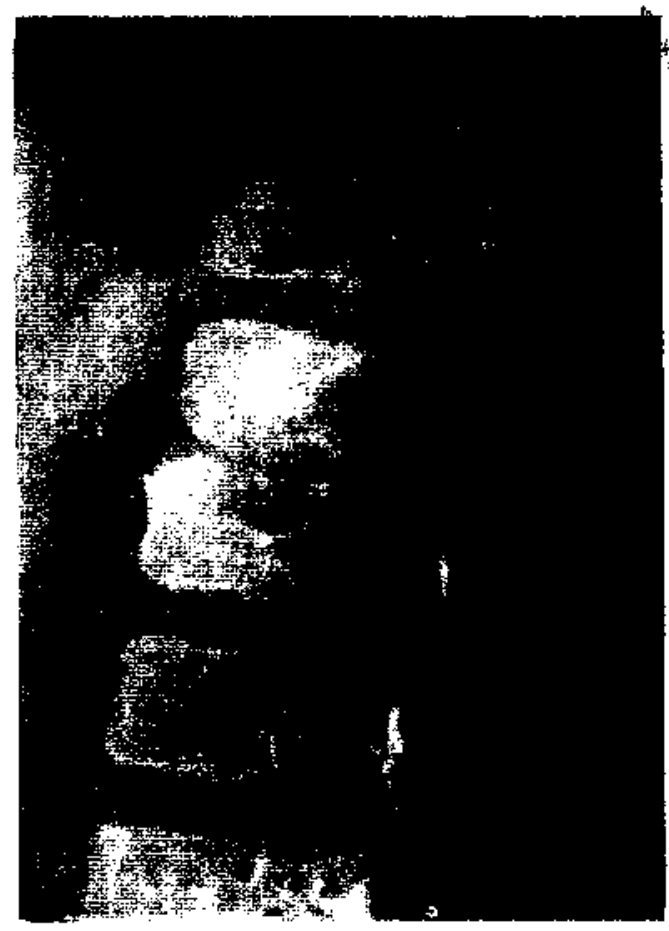

B

A: Dicientbre 1952. B: Mayo 1953.

B. Locales: En todos los casos en que existían abscesos o fístulas, estas mejoraron en un plazo inferior a 45 . Hubo necesidad de inyección local de la droga y aspiración del pus, en el caso $\mathrm{N}$ \% 7 una osteítis del hueso frontal, en que el cultivo del material extraído dió desarrollo de Microbacterium tuberculosis. Se usó una solución de $\mathbf{2 5}$ mgrs. de hidrazida en un centímetro cúbico de suero fisiológico, día por medio, con un total de 4 inyecciones, con lo que se produjo una total reabsorción del absceso en 25 días y la lesión ósea radiológica curó en 10 meses.

Pero indudablemente su acción más notable fué sobre las molestias ? reacciones locales, especialmente dolores $y$ por lo tanto sobre la impotencia funcional. Esta acción se manifestó ya al mes de iniciada la cura, o aun antes.

Los controles radiográficos periódicos nos demostraron una rápida tendencia a la reconstrucción ósea, podriamos decir una detención en la evolución del proceso destructivo y su consecuencia, la cicatrizacjón manifiesta. Así, en el caso No 3 . en que no alcanzó a producirse lesión ósea visible costeoartritis de la rodilla de for- 
ma sinovial) hasta la fecha no ha aparecido.

Cuando la destrucción ósea fué apreciable, la reconstrucción demostrable a la radiografía, se realizó con el ritmo y en la misma manera que el esqueleto normalmente se repara, por lo tanto quedando secuelas de deformaciones, como en el Mal de Pott o en las coxitis, como correspondía a las características anatómicas y funcionales de la zona en que actuó el proceso. De ahí que los resultados funcionales, en estos casos, aparezcan como mejores en las extremidades superiores que en las inferiores.

En todos los casos se apreció esta notable tendencia a la curación. En ninguno el proceso dió la impresión de seguir evolucionando, ni mucho menos agravándose. En los Mal de Pott, en que existían discretos signos de irritación o compresión medular, estos desaparecieron rápidamente, sin que se produjeran paraplegias.

Los enfermos que interrumpieron la cura al poco tiempo de iniciada, en vista del alivio experimentado, volvieron con el proceso en clara reagudización.

Los abscesos fríos que fueron diagnosticados, desaparecieron sin dejar señales radicgráficas de calcificación.

\section{RESUMEN}

Se expone el resultado obtenido en 14 nin̄os con tuberculosis osteoarticular, de diversas localizaciones, tratados con hidrazida del ácido isonicotínico y controlados por un plazo mayor de un año. La droga fué administrada por vía bucal en dasis de $10 \mathrm{mgrs}$. por $\mathrm{kg}$.-día durante los 6 primeros meses y de $5 \mathrm{mgrs}$. por $\mathrm{kg}$.día en los 6 meses siguientes. Localmente se usó sólo en 1 caso en solución de $25 \mathrm{mgrs}$. por cc. de suero fisiológico. No se hizo ningún otro tratamiento médico ni quirúrgico.

Los resultados generales y locales fueron ampliamente satisfactorios en todos los casos. Hubo notable mejoría del estado nutritivo y disminución rápida de las manifestaciones de toxemia tuberculosa. Localmente se observó una rápida acción sobre los dolores y la curación de los abscesos y fístulas en un plazo menor de 45 días. La radiología demostró la detención del proceso destructivo, con tendencia rápida a la reparación del hueso.

\section{SUMMARY}

The results obtained in 14 children with osteoarticular tuberculosis of diverse localizations, treated with hidrazide of the isonicotinic acid and controled during more than one year, are detailed. The drug was given by mouth in doses of 10 mgrs. per kg.-day during the first 6 months and 5 mgrs. per kg.-day in the 6 months that followed. Locally it was only used in one case in a solution of 25 mgrs. per c.c. of saline solution. No other medical or surgical treatment was used.

The general and local results were very satisfactory in all cases. There was considerable improvement in the nutritional status and a quick decline of the tbc. toxemic manifestations. Locally, a rapid action was observed on the pains and the healing of abscesses and fistulas in a period of less than 45 days. $X$ rays showed a detention of the destructive processes with a quick tendency to healing of the bone. 